\title{
Vascularite à IgA (purpura rhumatoïde) de l'adulte
}

\section{Adult IgA Vasculitis (Henoch-Schönlein Purpura)}

\section{E. Pillebout}

Reçu le 17 janvier 2018; accepté le 19 mars 2018

(C) SRLF et Lavoisier SAS 2018

Résumé La vascularite à $\operatorname{IgA}(\mathrm{VIgA})$, anciennement purpura rhumatoïde (PR), est une vascularite systémique des petits vaisseaux à dépôts d'immunoglobulines $\mathrm{A}(\mathrm{IgA})$. Elle est beaucoup plus fréquente chez l'enfant que chez l'adulte (150 à 200 pour 1). La prévalence du PR chez l'adulte n'est pas connue et son incidence est estimée à $1 /$ million. La maladie de l'adulte semble en effet différer de celle de l'enfant par l'incidence et la gravité des manifestations cliniques. La VIgA est caractérisée par l'association d'un purpura vasculaire cutané à des signes articulaires et gastro-intestinaux. Une atteinte rénale s'associe parfois à ces signes. Il s'agit alors d'une glomérulonéphrite à dépôts mésangiaux d'IgA. Plus rarement des localisations neurologiques, pulmonaires, cardiaques ou urogénitales peuvent s'observer. Sa physiopathologie demeure inconnue, mais les IgA joueraient un rôle central. Lorsque la symptomatologie est peu sévère, seul un traitement symptomatique est conseillé. À l'opposé, dans les formes digestive ou rénale sévères, des traitements plus agressifs associant, le plus souvent, des corticostérö̈des à du cyclophosphamide ont été proposés. Leur efficacité est en cours d'évaluation. Le pronostic à court terme de la maladie dépend de la sévérité de l'atteinte digestive, et à long terme de la sévérité de l'atteinte rénale. Les études ayant un suivi suffisamment prolongé montrent qu'un tiers des malades adultes évoluent vers l'insuffisance rénale terminale.

Mots clés Vascularite à IgA · Vascularite des petits vaisseaux $\cdot$ Purpura vasculaire $\cdot$ Néphropathie à $\operatorname{IgA}$. Adulte

Abstract IgA vasculitis (IgAV) is a systemic IgA vasculitis affecting small vessels. IgAV usually affects children; it is

E. Pillebout $(\bowtie)$

Service de néphrologie et de transplantation, hôpital Saint-Louis, 1, avenue Claude-Vellefaux, F-75010 Paris, France

e-mail : evangeline.pillebout@aphp.fr

Inserm 1149, CRI, faculté de médecine Xavier-Bichat, 16, rue Henri-Huchard, F-75018 Paris, France rare in adults (150-200 for 1). The disease is often more serious in adults with more frequent and severe nephritis. Prevalence of adult IgAV is unknown and its annual incidence is 1 in 1 million. The dominant clinical features include cutaneous purpura, arthritis, and gastrointestinal symptoms. Sometimes nephritis occurs, typically as glomerulonephritis with IgA mesangial deposits. Pulmonary, cardiac, genital, and neurological symptoms have also been observed. Although the cause is unknown, it is clear that IgA plays a pivotal role in the immunopathogenesis of IgAV. Only symptomatic treatment is advised in case of selflimited disease. Treatment of severe IgAV, nephritis, or gastrointestinal manifestations, is not established but some studies, which need to be confirmed, reported the benefit of corticosteroids combined with immunosuppressive drugs. Short-term outcome depends on the severity of the gastrointestinal manifestations. The long-term prognosis is heavily dependent on the presence and severity of nephritis. Studies with prolonged follow-up show up to one third of adult patients reaching end stage renal failure.

Keywords IgA vasculitis · Small vessel vasculitis · Purpura * IgA glomerulonephritis · Adults

\section{Introduction}

La vascularite à $\operatorname{Ig} \mathrm{A}$ (VIgA-purpura rhumatoïde ou syndrome de Schönlein-Henoch) est une vascularite systémique des petits vaisseaux en rapport avec des dépôts tissulaires de complexes immuns contenant des immunoglobulines A (IgA). Il atteint principalement l'enfant, l'affection étant plus rare chez l'adulte. La maladie est caractérisée par l'association de signes cutanés, articulaires et gastro-intestinaux pouvant survenir par poussées. Une atteinte rénale s'associe parfois à ces signes. Le pronostic de la maladie à court terme dépend de la sévérité de l'atteinte digestive, et à long terme de l'atteinte rénale. La fréquence et le pronostic de cette atteinte sont extrêmement variables selon les séries. Des 
publications récentes montrent l'évolution vers l'insuffisance rénale (IR) chronique parfois plus de dix ans après la première poussée [1-3].

\section{Épidémiologie}

La VIgA peut se manifester à tout âge (de 5 mois à 89 ans) mais atteint principalement l'enfant entre 3 et 15 ans. Chez l'enfant, son incidence est de l'ordre de $1 / 6660$ à $1 / 4$ 880 enfants par an [4]. Elle est beaucoup plus rare chez l'adulte où son incidence serait de l'ordre de 1 pour 1 million $[5,6]$. Le ratio enfants/adultes varierait ainsi de 150 à 205 . La VIgA de l'adulte diffère de celle de l'enfant par sa faible incidence et la plus grande sévérité des manifestations cliniques $[3,7-10]$. La maladie est plus fréquente chez les patients de sexe masculin (sex-ratio : 1,5). L'incidence augmente en hiver mais peut varier d'une année à l'autre. La VIgA est rapportée dans tous les pays du monde, mais sa distribution est variable. Elle paraît plus fréquente au Japon, en Asie du Sud-Est, en Europe et en Australie qu'en Amérique du Nord et en Afrique du Sud. On l'observe dans toutes les ethnies mais est plus rare chez les sujets de race noire.

\section{Description anatomoclinique}

\section{Critères diagnostiques}

Le diagnostic de la VIgA repose sur l'association de signes cliniques extrêmement évocatrice. Il s'agit de l'association d'un purpura vasculaire cutané à des manifestations articulaires, digestives et rénales. Il n'existe aucun signe biologique spécifique de la maladie. Le taux sérique d'IgA est élevé dans $60 \%$ des cas, mais cela ne constitue en aucun cas un argument formel pour affirmer le diagnostic. L'histologie cutanée (vascularite leucocytoclasique) et/ou rénale (glomérulonéphrite endocapillaire proliférative) associées à la présence de dépôts d'IgA dans ces tissus peuvent être utiles, surtout chez l'adulte. Les critères les plus récents sont ceux d'EULAR/PRINTO/PRES : chez un enfant ayant un purpura ou des pétéchies prédominant aux membres inférieurs, le diagnostic peut être fait si au moins un des quatre critères suivants est présent :

- douleurs abdominales ;

- présence d'IgA à l'histologie ;

- arthrite ou arthralgie ;

- atteinte rénale [11].

Ces critères sont néanmoins difficilement applicables à l'adulte, l'âge étant un des critères principaux.

\section{Diagnostic différentiel}

Les autres causes de purpura sont éliminées : thrombopénie $(<10 \mathrm{~g} / \mathrm{l})$, hémopathie ou maladie infectieuse, tant bactérienne que virale. À l'opposé des formes pédiatriques chez l'adulte, le purpura rhumatoïde (PR) est loin d'être la première cause de vascularite. Les autres maladies systémiques responsables de purpura sont évoquées de principe : vascularites à anticorps anticytoplasme des polynucléaires neutrophiles, de Churg et Strauss, lupus érythémateux disséminé, cryoglobulinémie mixte, etc. L'histologie, notamment cutanée, permet le plus souvent de distinguer les différentes vascularites. Enfin, la vascularite cutanée d'hypersensibilité induite par les médicaments est parfois difficile à distinguer lorsque la VIgA se présente dans sa forme cutanée isolée.

\section{Atteintes extrarénales}

Les signes généraux sont fréquents mais le plus souvent modérés. La fièvre, généralement peu élevée est présente chez la moitié des patients. Un syndrome inflammatoire biologique est fréquent, mais généralement modéré.

- L'atteinte cutanée est quasiment constante et inaugure le tableau clinique dans plus de deux tiers des cas. Il s'agit le plus souvent d'un purpura vasculaire (Fig. 1). Il est symétrique, prédomine aux zones de pression, en particulier autour des chevilles et au niveau des fesses mais peut s'étendre à l'ensemble du tégument. La lésion primitive est généralement une pétéchie, elle peut confluer pour former des macules, voire des ecchymoses. Chez l'adulte elle se complique de nécrose ou de bulles hémorragiques dans 5 à $35 \%$ des cas, qui sont exceptionnelles chez l'enfant $[3,12]$. Les lésions régressent progressivement pour disparaître en 15 jours. Il peut n'y avoir qu'un seul rash de résolution rapide ou plusieurs poussées successives. L'étude de la biopsie cutanée montre, typiquement, une vascularite leucocytoclasique avec nécrose fibrinoïde et infiltrat périvasculaire de neutrophiles et de cellules mononucléées. En immunofluorescence, on observe dans la paroi des vaisseaux lésés des dépôts d'immunoglobulines $\mathrm{A}$, de la fraction $\mathrm{C} 3 \mathrm{du}$ complément et de fibrine ;

- les manifestations articulaires sont présentes dans deux tiers des cas et se traduisent par des arthralgies touchant principalement les chevilles et les genoux. Elles sont d'intensité variable, souvent rapidement résolutives. Une ou plusieurs articulations sont touchées, simultanément ou successivement. À la douleur peut s'associer un gonflement périarticulaire en rapport le plus souvent avec une synovite qui ne détruit jamais l'articulation ;

- les manifestations digestives sont fréquentes, variables en fonction des séries [13]. Il s'agit de douleurs spasmodiques, modérées (86 \%) mais pouvant être sévères, 


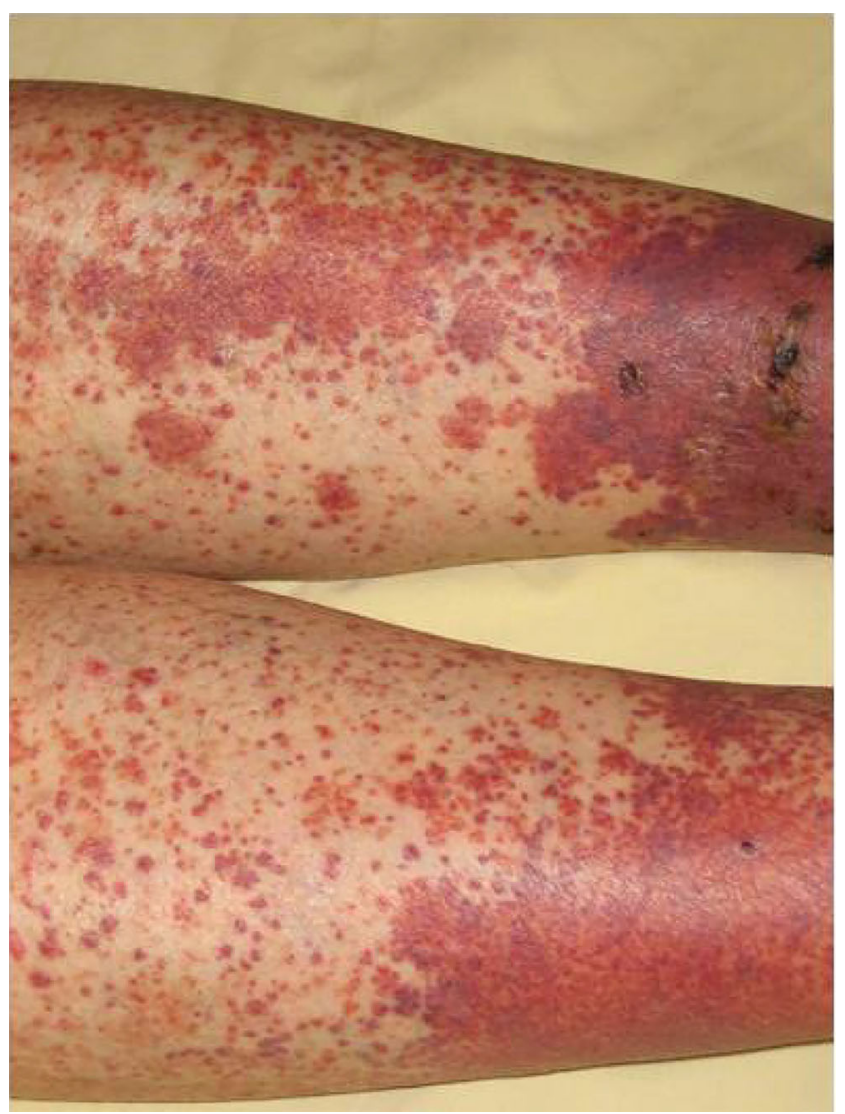

Fig. 1 Vascularite à $\operatorname{IgA}$, lésions cutanées Purpura vasculaire des membres inférieurs, symétrique, déclive, prédominant aux chevilles. Lésions nécroticobulleuses et éléments purpuriques de plus petite taille

conduisant alors à la laparotomie. Elles peuvent s'associer à une hémorragie digestive occulte (66\%) [présence de sang dans les selles à la bandelette] mais parfois gravissime (20\%), engageant le pronostic vital $[14,15]$. L'échographie et/ou le scanner abdominal (Fig. 2A) sont utiles en mettant en évidence une paroi intestinale épaissie avec une infiltration inflammatoire du mésentère en regard des hématomes sous-muqueux ou pariétaux parfois sténosants et en décelant une dilatation segmentaire d'une anse. L'invagination intestinale se voit plutôt chez le jeune enfant. L'endoscopie digestive haute et basse permet un diagnostic précis topographique des lésions, de leur extension, et d'orienter les biopsies digestives. Les lésions prédominent dans la deuxième portion du duodénum. Elle montre un érythème de la muqueuse avec purpura pétéchial, des érosions, voire de véritables plages de nécrose de la paroi digestive (Fig. 2B);

- les autres manifestations sont beaucoup plus rares, voire exceptionnelles. Dans le cadre des manifestations neurologiques $(<1 \%$ ), on peut observer des céphalées (manifestation la plus fréquente de cette atteinte extrarénale
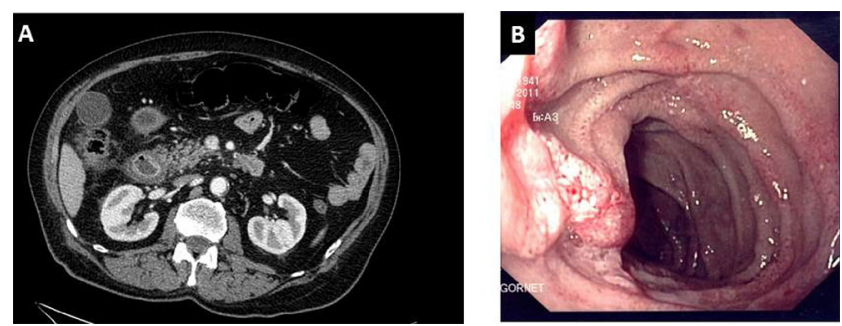

Fig. 2 Vascularite à $\operatorname{IgA}$, atteinte digestive Scanner digestif : aspect infiltré, épaissi et œdémateux d'une anse grêle iléale avec infiltration de la graisse mésentérique (A). Purpura de la muqueuse gastrique (B) visualisé par endoscopie

exceptionnelle), des convulsions, des parésies, voire un coma en rapport alors avec des lésions ischémiques ou thrombotiques secondaires à la vascularite cérébrale pouvant être objectivées par l'IRM [16,17]. Quelques cas ont été rapportés chez l'enfant de PRES (Posterior Reversible Encephalopathy) syndrome, toujours dans un contexte d'hypertension artérielle non équilibrée. Plus exceptionnellement sont décrites des orchiépididymites (le plus souvent chez le jeune garçon), urétrites, pancréatites, parotidites, myosites, épisclérites, hémorragies intraalvéolaires [18-20] et myocardites.

\section{L'atteinte rénale}

Une atteinte rénale survient dans 20 à $54 \%$ des cas de VIgA chez l'enfant et dans 45 à $85 \%$ chez l'adulte [9,12,21-24]. L'incidence varie selon les séries, en fonction du mode de recrutement des malades et des critères diagnostiques retenus pour affirmer l'atteinte rénale. Chez l'adulte, l'atteinte rénale de la $\mathrm{VIgA}$ ne représenterait que 0,6 à $2 \%$ des néphropathies [25].

L'atteinte rénale survient généralement au cours du premier mois de la maladie, mais des anomalies du sédiment urinaire peuvent être observées après plusieurs mois, parfois au cours d'une nouvelle poussée de purpura.

L'hématurie, le plus souvent microscopique, est le signe le plus précoce. À cette hématurie peuvent s'associer d'autres signes évocateurs de glomérulonéphrite, une protéinurie de débit variable, voire un syndrome néphrotique et une insuffisance rénale. Chez l'adulte, l'atteinte rénale est non seulement plus fréquente, mais également plus sévère. La présence d'une insuffisance rénale au moment du diagnostic est exceptionnelle chez l'enfant, alors que son incidence peut atteindre $32 \%$ chez l'adulte $[3,10,26]$. Une hypertension artérielle peut s'associer à ces signes ou être isolée.

La néphropathie de la VIgA est une néphropathie glomérulaire à dépôts d'IgA très proche de la néphropathie à $\operatorname{IgA}$ (NIgA-maladie de Berger). Seul le contexte clinique permet d'ailleurs de les distinguer sur le plan histologique [27,28]. 
Sauf si la biopsie rénale est nécessaire au diagnostic de la vascularite, elle n'est généralement indiquée, comme au cours de la NIgA, que s'il existe une protéinurie significative ou une altération de la fonction rénale. L'étude en immunofluorescence confirme le diagnostic de glomérulonéphrite à dépôts d'IgA (Fig. 3A). Les dépôts mésangiaux sont présents dans tous les glomérules, leur abondance est variable, et ils peuvent même déborder le long des parois des capillaires glomérulaires voire du pôle vasculaire. À ces dépôts d'IgA (essentiellement IgA1 et lambda plus fréquemment que kappa) s'associent constamment des fractions $\mathrm{C} 3 \mathrm{du}$ complément, parfois des dépôts d'IgG ( $40 \%$ des cas) et plus rarement d'IgM (20\% des cas) [29]. Les dépôts mésangiaux observés dans les glomérules scléreux permettent un diagnostic rétrospectif. L'examen en microscopie optique montre une grande diversité de type et de sévérité de lésions glomérulaires. Nous avons proposé pour l'adulte une classification basée sur une forte corrélation anatomoclinique [3]. Elle distingue :

- la glomérulonéphrite " mésangiopathique »;

- la glomérulonéphrite segmentaire et focale (Fig. 3B) ;

- la glomérulonéphrite proliférative endocapillaire diffuse (Fig. 3C) ;

- la glomérulonéphrite endocapillaire et extracapillaire ;

- enfin, le rein fibreux, correspondant au stade terminal.

Chez l'adulte, la lésion la plus fréquemment rencontrée est la classe 3. La prolifération endo- et extracapillaire est rare, touchant moins de $10 \%$ des cas. On peut également observer des lésions moins spécifiques comme la nécrose fibrinoïde qui s'observe surtout à la phase aiguë. Des lésions tubulo-interstitielles sont fréquemment associées aux lésions glomérulaires et sont fortement corrélées à la présence d'une insuffisance rénale. Il existe volontiers des cylindres hématiques. Les lésions vasculaires sont également fréquentes, même chez l'adulte jeune. Comme au cours de la NIgA, une nouvelle classification histologique sur le modèle de la classification d'Oxford va être établie dans une large cohorte
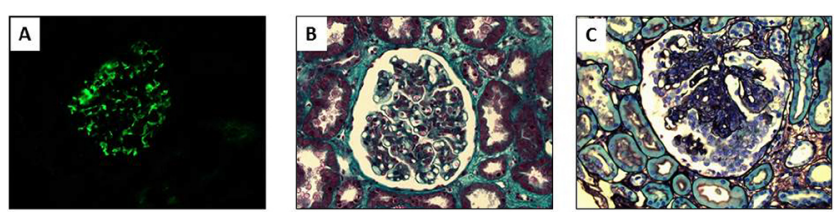

Fig. 3 Purpura rhumatoïde, histologie rénale Immunofluorescence : dépôts granuleux, mésangiaux d'IgA (A) $\times 400$ Microscopie optique. B. Glomérulonéphrite segmentaire et focale (classe 2) au contact d'un dépôt fibrinoïde. Cylindre hématique. Trichrome de Masson $\times$ 400. C. Glomérulonéphrite endocapillaire diffuse et sévère (classe $3 b$ ). Foyer de nécrose fibrinoïde et petite réaction proliférative extracapillaire en croissant. Trichrome de Masson $\times 200$ internationale de patients pédiatriques et adultes [30,31]. Son but est avant tout pronostique et permettrait, si elle est unanimement admise, d'homogénéiser les cohortes de patients des futures études. Récemment, des néphrologues et pathologistes de dix pays différents se sont réunis et, après avoir étudié l'histoire clinique et la biopsie rénale de 265 patients du monde entier atteints de NIgA, ont proposé une nouvelle classification, dite d'Oxford. Cette nouvelle classification, simple d'utilisation, serait très reproductible et bien corrélée au pronostic clinique. En prenant en compte les lésions glomérulaires, tubulo-interstitielles et vasculaires, les auteurs suggèrent que seules l'hypercellularité mésangiale et endocapillaire, les lésions de glomérulosclérose focale et la fibrose interstitielle/atrophie tubulaire sont des facteurs pronostiques. Son intérêt comme facteur pronostique, indépendamment de la protéinurie, de la pression artérielle et du débit de filtration glomérulaire estimé, a été validé par d'autres cohortes.

\section{Étiologie/physiopathologie}

La physiopathologie de la VIgA est à ce jour peu connue. La plupart des études se concentrent sur la NIgA considérée comme la même pathologie sans ses manifestations systémiques. La NIgA, bien plus fréquente, a donc été beaucoup plus étudiée, et des progrès considérables ont été faits ces dernières années.

L'IgA joue, au cours de ces deux pathologies, un rôle central. La VIgA est, par définition, caractérisée par des dépôts intratissulaires (mésangiaux ou vasculaires) d'IgA. $\mathrm{Au}$ cours des trois premiers mois de la maladie, on note chez la moitié des patients une augmentation du taux sérique des IgA, déséquilibré en faveur des sous-classes IgA1, plutôt polymériques et retrouvés dans des complexes immuns circulants. Il existe par ailleurs des anomalies de la glycosylation de ces IgA, notamment du contenu en galactose et en acide sialique. Ces anomalies quantitatives et structurales des IgA modifieraient la liaison à leurs récepteurs, le $\mathrm{RFcI}$ (CD89) exprimé par les monocytes circulants et le récepteur de la transferrine (CD71) présent sur les cellules mésangiales. Ces IgA anormales induisent la libération, dans la circulation, de CD89 soluble qui participe à la formation des complexes circulants contenant des IgA. Ces complexes se déposent secondairement dans le mésangium rénal en se fixant au CD71, surexprimé par les cellules mésangiales des patients. Ils induiraient alors la prolifération des cellules mésangiales et l'inflammation locale responsable de l'insuffisance rénale progressive (Fig. 4). La récente étude prospective du groupe HSPronostic a montré que les anomalies de la glycosylation des IgA et l'augmentation du taux de complexes IgA-CD89 et IgA-IgG sont associés à la présence 


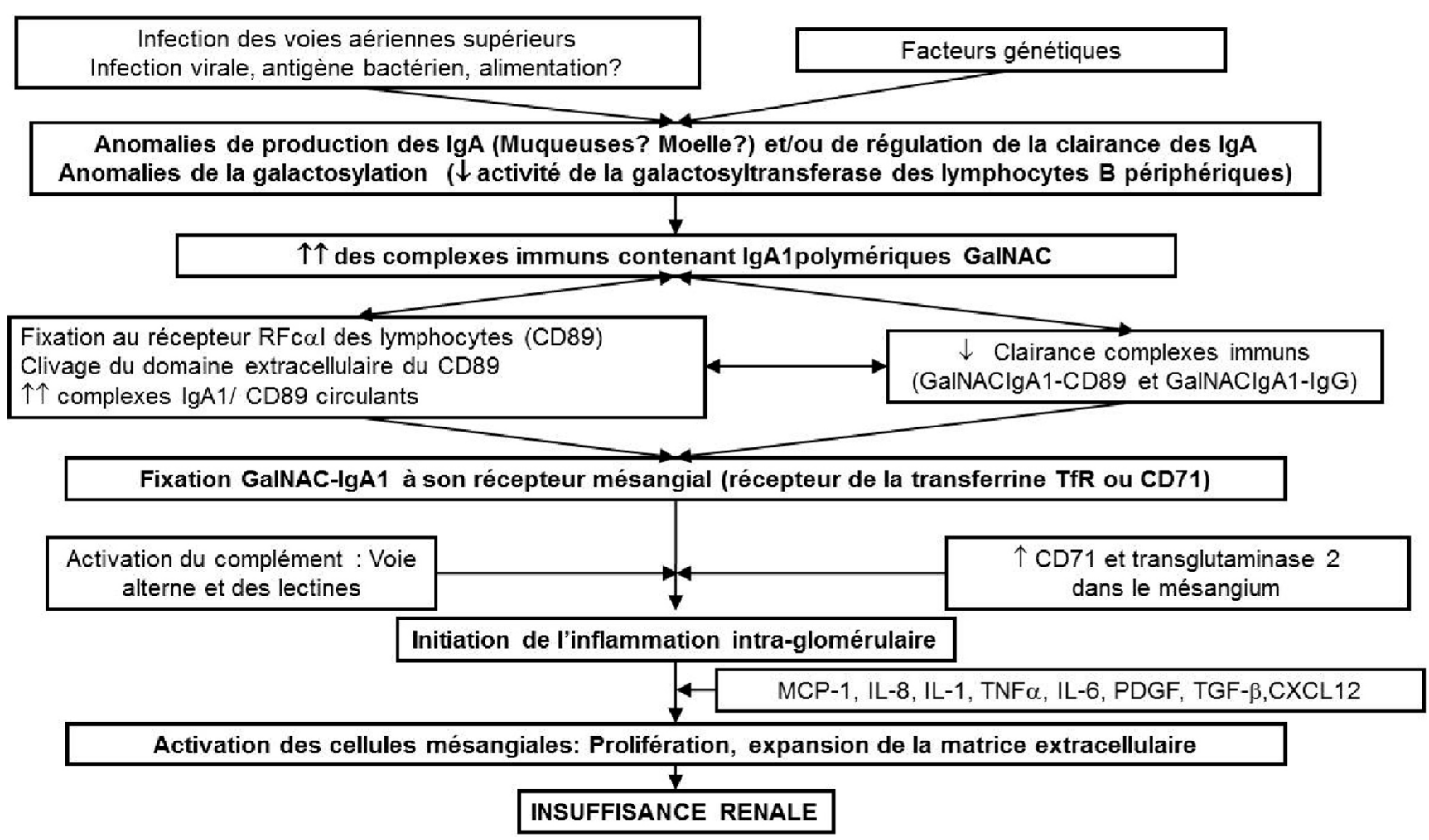

Fig. 4 Physiopathologie de la néphropathie à $\operatorname{IgA}$

de la néphropathie, confirmant le fait que la VIgA et la NIgA partagent le même mécanisme physiopathologique [10,26].

Que ce soit au cours de la NIgA ou de la VIgA, la maladie est souvent précédée d'une infection de la sphère ORL ou respiratoire (Streptocoque, adénovirus, parvovirus, Mycoplasma pneumoniae, etc.) ou d'une prise médicamenteuse, toxique ou alimentaire, en particulier chez l'enfant. Elle a également été associée à d'autres infections virales telles que celles à parvovirus B19, EBV, CMV, HIV $[21,23]$. Plus récemment, il a été montré chez l'adulte l'association à certains cancers, et particulièrement des épithéliums muqueux, plus fréquents au cours de l'intoxication alcoolotabagique (tumeurs des voies aérodigestives supérieures et pulmonaires), sans que l'existence d'un véritable syndrome paranéoplasique puisse être affirmée [32].

\section{Évolution}

Chez l'enfant, la VIgA se manifeste le plus souvent par une poussée unique. Chez l'adulte, $22 \%$ des patients auront plusieurs poussées, et $33 \%$ des adultes passeront à la chronicité.

Le risque vital est avant tout lié à l'atteinte digestive, lorsque celle-ci se complique de perforation ou d'hémorragie gastro-intestinale non contrôlée. Ces complications, plus fréquentes chez l'enfant, sont néanmoins exceptionnelles. L'atteinte pulmonaire (hémorragie intra-alvéolaire) est très rare mais souvent fatale $[19,20,33]$. Après une extraction de la base de données Pubmed, des auteurs [19] ont décrit 36 patients (28 publications), estimant la prévalence entre 0,8 et $5 \%$. L'hémoptysie ( $75 \%$ ), l'anémie (74 \%) et l'infiltration à l'imagerie (94\%) sont les symptômes de l'hémorragie intra-alvéolaire chez ces patients. Une biopsie pulmonaire a été pratiquée chez $36 \%$ des patients, objectivant une vascularite leucocytoclasique dans $69 \%$ des cas, IgA positive dans la moitié des cas. La mortalité est de 27,8\%. Chez les patients se présentant avec un tableau sévère associant une atteinte rénale et pulmonaire, il faut penser à rechercher les ANCA. En effet, une VIgA avec IgA-ANCA doit être prise en charge comme une vascularite à ANCA. Le pronostic à long terme dépend essentiellement de l'évolution de l'atteinte rénale.

Le risque d'évolution vers l'insuffisance rénale terminale nécessitant la dialyse chez l'enfant est variable de 2,5 à $25 \%$, mais en moyenne de l'ordre de $8 \%$. En Europe, $3 \%$ des enfants dialysés le sont en raison d'une VIgA. Deux études, concernant des enfants suivis plus de 20 ans après le diagnostic de VIgA, donnent un recul suffisant vis-à-vis de cette pathologie $[1,34]$. Elle souligne la nécessité d'un suivi néphrologique prolongé. En effet, même les enfants considérés en rémission dix ans après le diagnostic ont développé 20 ans plus tard une hypertension artérielle ou une insuffisance rénale chronique. Cette surveillance est indispensable en cas de grossesse survenant chez des patientes 
ayant des antécédents de VIgA. Un tiers des grossesses de la première série et $70 \%$ de la deuxième sont compliquées d'hypertension artérielle gravidique ou toxémique et jusqu'à $16 \%$ de mort fotale [1].

Chez l'adulte, le risque de développer une insuffisance rénale chronique est fréquent, de 8 à $68 \%$, en moyenne de l'ordre de $18 \%$. Ces différences d'incidences sont dues à de nombreux facteurs : petites séries de patients, grande discordance d'âge d'une étude à l'autre, mode de recrutement (avec ou sans atteinte rénale) et recul des études, etc.

\section{Facteurs pronostiques}

La plupart des études, que ce soit chez l'enfant ou chez l'adulte, s'accordent à dire qu'il n'existe aucune corrélation entre la présentation ou l'intensité des signes extrarénaux et l'histologie ou l'évolution de l'atteinte rénale.

Chez l'enfant, il semble exister une assez bonne corrélation entre la présentation clinique néphrologique, l'examen histologique rénal et l'évolution rénale. Néanmoins, l'interprétation des études est difficile en raison de la possible influence des traitements, le plus souvent hétérogènes et mal précisés dans le texte. La présence ou la persistance d'un ratio protéine/créatinine urinaire à plus de $1 \mathrm{~g} / \mathrm{g}$ et/ou d'une insuffisance rénale au diagnostic et la présence, sur la biopsie rénale, de plus de $50 \%$ de croissants ou d'une fibrose interstitielle sont des facteurs de risque importants de l'évolution vers l'insuffisance rénale, tant chez l'adulte que chez l'enfant. Aucune dysfonction rénale n'est observée chez les enfants qui n'ont jamais eu d'anomalie urinaire pendant les six premiers mois. Aucune étude n'est disponible chez l'adulte pour affirmer une telle évolution. Il est donc recommandé de surveiller le sédiment urinaire au moins six mois chez tout patient pour lequel le diagnostic de VIgA sans atteinte rénale a été porté et de manière plus prolongée encore s'il existe une atteinte rénale.

Toutes ces études décrivent néanmoins l'évolution spontanément favorable de patients ayant une forme clinique et histologique initiale sévère et à l'opposée l'évolution vers l'insuffisance rénale chronique de patients avec une symptomatologie rénale initiale minime, ce qui complique considérablement les décisions thérapeutiques et l'élaboration des études cliniques. Les patients sont en effets exposés d'un côté au risque d'effets secondaires de traitements inutiles et de l'autre côté au risque de dégradation de fonction rénale s'ils sont insuffisamment traités. Identifier précocement des biomarqueurs pronostiques est un enjeu majeur de la recherche. L'étude prospective du groupe HSPronostic a récemment montré que le taux d'IgA urinaire au diagnostic était, chez l'adulte, corrélé au pronostic rénal à un an plus que la protéinurie ou la fonction rénale mesurée à la phase aiguë de la maladie [26].

\section{Le traitement}

\section{Le traitement symptomatique}

Le repos au lit limite l'extension du purpura cutané mais n'influence en rien l'évolution de l'atteinte digestive ou rénale. Il doit être limité aux patients ayant des douleurs articulaires telles que la mobilisation est difficilement envisageable.

Les antalgiques, au premier rang desquels le paracétamol et ses dérivées, sont efficaces sur les douleurs articulaires et musculaires. Les anti-inflammatoires non stéroïdiens sont réservés aux douleurs articulaires résistantes aux antalgiques simples et sont à éviter chez les patients avec atteinte rénale et/ou, en raison de leur effet antiagrégant plaquettaire, chez les patients ayant un saignement digestif actif. Les antispasmodiques peuvent soulager les patients souffrant de douleurs abdominales.

Enfin, les mesures de néphroprotection sont recommandées chez tout patient ayant une atteinte rénale. Un bloqueur du système rénine-angiotensine (inhibiteur de l'enzyme de conversion, antagoniste du récepteur de l'angiotensine II) doit être utilisé en première intention pour obtenir un contrôle optimal de la pression artérielle et du débit de protéinurie. Un suivi spécialisé est nécessaire tant qu'il persiste des anomalies cliniques ou biologiques. Puis, s'il n'existe plus aucune anomalie urinaire, que la fonction rénale est normale et la pression artérielle contrôlée, un suivi annuel est recommandé par le médecin traitant par exemple.

Ces mesures symptomatiques sont généralement suffisantes chez la plupart des patients. Des traitements plus spécifiques ont été proposés aux patients ayant une forme clinique préoccupante. Nous avons proposé récemment un arbre décisionnel (Fig. 5).

\section{Les traitements spécifiques}

Les corticostéroïdes sont efficaces pour diminuer les douleurs abdominales et articulaires, comme les antalgiques usuels. Ils sont parfois utilisés en cas d'atteinte digestive sévère, après échec de l'alimentation parentérale exclusive, avec stricte surveillance médicochirurgicale. Il n'y a pas d'effet préventif de la corticothérapie sur l'atteinte rénale [35].

Chez les enfants présentant une néphropathie sévère, le traitement doit être discuté. Les résultats des études utilisant les stéroïdes seuls sont contradictoires et difficiles à interpréter, car la plupart sont faites en l'absence de groupe témoin et/ou sont rétrospectives. Les perfusions de méthylprednisolone relayées par une courte corticothérapie par voie orale (comme pour le traitement de la NIgA selon le schéma thérapeutique proposé par Locatelli $[36,37])$ est le traitement le plus souvent prescrit par les pédiatres et recommandé chez l'adulte également si la protéinurie reste 

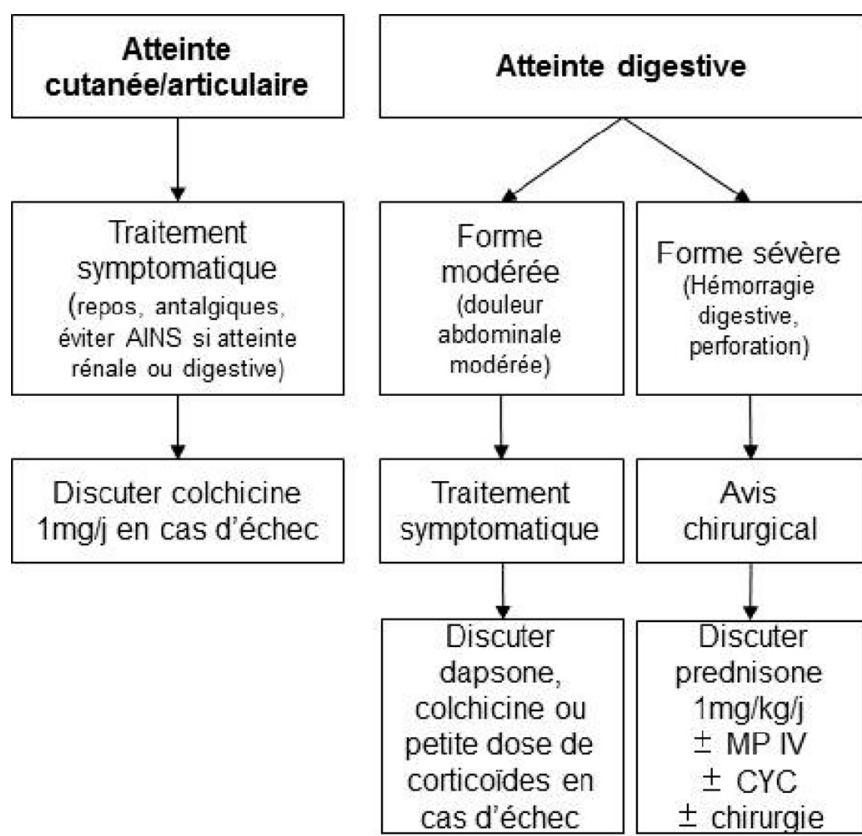

Fig. 5 Prise en charge thérapeutique

supérieure à $1 \mathrm{~g} / \mathrm{g}$ de créatininurie malgré au moins trois mois de bloqueurs du système rénine-angiotensine [40]. Le schéma consiste chez l'adulte en : méthylprednisolone intraveineux, $1 \mathrm{~g} / \mathrm{j}$ trois jours de suite à renouveler deux et quatre mois plus tard avec dans l'intervalle de la prednisolone per os à la posologie de $0,5 \mathrm{mg} / \mathrm{kg}$ un jour sur deux et chez l'enfant, bolus de méthylprednisolone $1 \mathrm{~g} / 1,73 \mathrm{~m}^{2} \times 3$ puis prednisone $2 \mathrm{mg} / \mathrm{kg}$ par jour $\times 1$ mois puis $2 \mathrm{mg} / \mathrm{kg}$ par deux jours $\times 1$ mois puis arrêt sur un mois.

Des traitements plus agressifs, associant aux stéroïdes du cyclophosphamide, n'ont pas démontré leur efficacité, tant chez l'enfant que chez l'adulte [35,38]. Des études sont en cours pour évaluer d'autres immunosuppresseurs : mycophénolate mofétil, anticalcineurine, rituximab, etc. Au cours de la NIgA, les résultats des essais prospectifs évaluant ces traitements sont décevants, mais les récents résultats d'une étude rétrospective concernant le rituximab au cours de la VIgA sont encourageants [39].

Néanmoins, chez les patients ayant une insuffisance rénale rapidement progressive et une glomérulonéphrite proliférative avec plus de $50 \%$ de croissant (GNRP), il est recommandé, comme au cours de la NIgA, de traiter les patients comme une vascularite à ANCA, avec des perfusions de méthylprednisolone relayées par une corticothérapie par voie orale associée au cyclophosphamide IV [40].

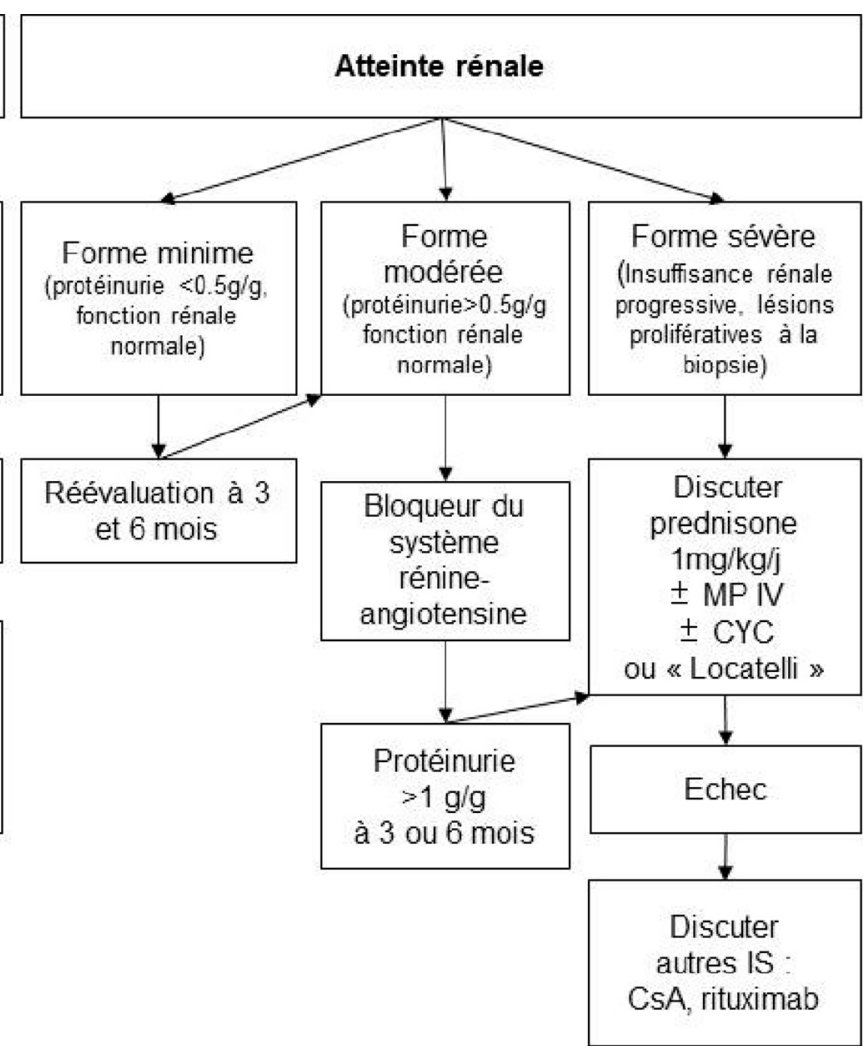

Prise en charge des formes pouvant engager le pronostic vital

Il est difficile de recommander une prise en charge des formes très sévères de VIgA puisqu'elles sont exceptionnelles. Il n'y a aucun essai clinique, seulement des cas isolés, rapportés qui suggèrent l'intérêt d'ajouter des immunoglobulines [41] ou des plasmaphérèses [8,42-44] aux stéroïdes intraveineux et aux immunosuppresseurs dans les atteintes digestives, pulmonaires, rénales, voire cutanées sévères.

\section{Conclusion}

La vascularite à $\operatorname{IgA}$ est une maladie rare de l'adulte. Les manifestations cliniques pouvant engager le pronostic vital et conduisant en réanimation sont exceptionnelles, digestives, pulmonaires, neurologiques et/ou rénales (syndrome de glomérulonéphrite rapidement progressive). Leur prise en charge thérapeutique n'est pas vraiment établie, mais l'association de stéroïdes intraveineux, d'immunosuppresseurs, voire de plasmaphérèses est le plus souvent prescrite. Le pronostic à long terme dépend de l'atteinte rénale.

Liens d'intérêts : l'auteur déclare ne pas avoir de lien d'intérêt. 


\section{Références}

1. Goldstein AR, White RH, Akuse R, Chantler C, (1992) Longterm follow-up of childhood Henoch-Schönlein nephritis. Lancet 339: 280-282

2. Ronkainen J, Nuutinen M, Koskimies O, (2002) The adult kidney 24 years after childhood Henoch-Schönlein purpura: a retrospective cohort study. Lancet 360: 666-670

3. Pillebout E, Thervet E, Hill G, Alberti C, Vanhille P, Nochy D, (2002) Henoch-Schönlein Purpura in adults: outcome and prognostic factors. J Am Soc Nephrol 13: 1271-1278

4. Gardner-Medwin JM, Dolezalova P, Cummins C, Southwood TR, (2002) Incidence of Henoch-Schönlein purpura, Kawasaki disease, and rare vasculitides in children of different ethnic origins. Lancet 360: 1197-1202

5. Watts RA, Lane S, Scott DG, (2005) What is known about the epidemiology of the vasculitides? Best Pract Res Clin Rheumatol 19: 191-207

6. Penny K, Fleming M, Kazmierczak D, Thomas A, (2010) An epidemiological study of Henoch-Schönlein purpura. Paediatr Nurs 22: $30-35$

7. Blanco R, Martinez-Taboada VM, Rodriguez-Valverde V, GarciaFuentes M, Gonzalez-Gay MA, (1997) Henoch-Schönlein purpura in adulthood and childhood: two different expressions of the same syndrome. Arthritis Rheum 40: 859-864

8. Kawasaki Y, Suzuki J, Murai M, Takahashi A, Isome M, Nozawa R, Suzuki S, Suzuki H, (2004) Plasmapheresis therapy for rapidly progressive Henoch-Schönlein nephritis. Pediatr Nephrol 19: 920-923

9. Garcia-Porrua C, Calvino MC, Llorca J, Couselo JM, GonzalezGay MA, (2002) Henoch-Schönlein purpura in children and adults: clinical differences in a defined population. Semin Arthritis Rheum 32: 149-156

10. Pillebout E, Jamin A, Ayari H, Housset P, Pierre M, Sauvaget V, Viglietti D, Deschenes G, Monteiro RC, Berthelot L, (2017) Biomarkers of IgA vasculitis nephritis in children. PLoS One 12: $\mathrm{e} 0188718$

11. Ozen S, Pistorio A, Iusan SM, Bakkaloglu A, Herlin T, Brik R, Buoncompagni A, Lazar C, Bilge I, Uziel Y, Rigante D, Cantarini L, Hilario MO, Silva CA, Alegria M, Norambuena X, Belot A, Berkun Y, Estrella AI, Olivieri AN, Alpigiani MG, Rumba I, Sztajnbok F, Tambic-Bukovac L, Breda L, Al-Mayouf S, Mihaylova D, Chasnyk V, Sengler C, Klein-Gitelman M, Djeddi D, Nuno L, Pruunsild C, Brunner J, Kondi A, Pagava K, Pederzoli S, Martini A, Ruperto N, (2010) EULAR/PRINTO/PRES criteria for Henoch-Schönlein purpura, childhood polyarteritis nodosa, childhood Wegener granulomatosis and childhood Takayasu arteritis: Ankara 2008. Part II: Final classification criteria. Ann Rheum Dis 69: 798-806

12. Audemard-Verger A, Terrier B, Dechartres A, Chanal J, Amoura Z, Le Gouellec N, Cacoub P, Jourde-Chiche N, Urbanski G, Augusto JF, Moulis G, Raffray L, Deroux A, Hummel A, Lioger B, Catroux M, Faguer S, Goutte J, Martis N, Maurier F, Riviere E, Sanges S, Baldolli A, Costedoat-Chalumeau N, Roriz M, Puechal X, Andre M, Lavigne C, Bienvenu B, Mekinian A, Zagdoun E, Girard C, Berezne A, Guillevin L, Thervet E, Pillebout E, (2017) Characteristics and management of $\operatorname{IgA}$ vasculitis (Henoch-Schönlein) in adults: data from 260 patients included in a French multicenter retrospective survey. Arthritis Rheumatol 69: $1862-1870$

13. Zhang Y, Huang X, (2008) Gastrointestinal involvement in Henoch-Schönlein purpura. Scand J Gastroenterol 43: 1038-1043

14. Kaswala D, Chodos A, Ahlawat S, (2016) Henoch-Schönlein Purpura With Gastrointestinal Involvement in an Adult Patient. Gastroenterol Hepatol (N Y) 12: 321-323
15. Yamazaki T, Akimoto T, Iwazu $\mathrm{Y}$, Sugase $\mathrm{T}$, Takeshima E, Numata A, Komada T, Yoshizawa H, Otani N, Morishita Y, Saito O, Takemoto F, Muto S, Kusano E, Nagata D, (2015) Henoch-Schönlein purpura complicated with severe gastrointestinal bleeding. CEN Case Rep 4: 106-111

16. Garzoni L, Vanoni F, Rizzi M, Simonetti GD, Goeggel Simonetti B, Ramelli GP, Bianchetti MG, (2009) Nervous system dysfunction in Henoch-Schönlein syndrome: systematic review of the literature. Rheumatology (Oxford) 48: 1524-1529

17. Lava SA, Peeters GG, Bianchetti MG, Goeggel Simonetti B, Simonetti GD, Milani GP, (2017) Posterior reversible encephalopathy syndrome in Henoch-Schönlein purpura. Rheumatol Int 37: 461-463

18. Rajagopala S, Parameswaran S, Ajmera JS, Ganesh RN, Katrevula A, (2017) Diffuse alveolar hemorrhage in IgA nephropathy: case series and systematic review of the literature. Int $\mathrm{J}$ Rheum Dis 20: 109-121

19. Rajagopala S, Shobha V, Devaraj U, D’Souza G, Garg I, (2013) Pulmonary hemorrhage in Henoch-Schönlein purpura: case report and systematic review of the english literature. Semin Arthritis Rheum 42: 391-400

20. Chen SY, Chang KC, Yu MC, Asueh S, Ou LS, (2011) Pulmonary hemorrhage associated with Henoch-Schönlein purpura in pediatric patients: case report and review of the literature. Semin Arthritis Rheum 41: 305-312 (doi: 10.1016/j.semarthrit.2011.03.007)

21. Rostoker G, (2001) Schönlein-henoch purpura in children and adults: diagnosis, pathophysiology and management. BioDrugs 15: $99-138$

22. Calvino MC, Llorca J, Garcia-Porrua C, Fernandez-Iglesias JL, Rodriguez-Ledo P, Gonzalez-Gay MA, (2001) Henoch-Schönlein purpura in children from northwestern Spain: a 20-year epidemiologic and clinical study. Medicine (Baltimore) 80: 279-290

23. Saulsbury FT, (1999) Henoch-Schönlein purpura in children. Report of 100 patients and review of the literature. Medicine (Baltimore) 78: 395-409

24. Trapani S, Micheli A, Grisolia F, Resti M, Chiappini E, Falcini F, De Martino M, (2005) Henoch Schönlein purpura in childhood: epidemiological and clinical analysis of 150 cases over a 5 -year period and review of literature. Semin Arthritis Rheum 35: $143-153$

25. Rieu P, Noel LH, (1999) Henoch-Schönlein nephritis in children and adults. Morphological features and clinicopathological correlations. Ann Med Interne (Paris) 150: 151-159

26. Berthelot L, Jamin A, Viglietti D, Chemouny JM, Ayari H, Pierre M, Housset P, Sauvaget V, Hurtado-Nedelec M, Vrtovsnik F, Daugas E, Monteiro RC, Pillebout E, (2018) Value of biomarkers for predicting immunoglobulin A vasculitis nephritis outcome in an adult prospective cohort. Nephrol Dial Transplant (2017 Nov 3. doi: 10.1093/ndt/gfx300. [Epub ahead of print])

27. Nakamoto Y, Asano Y, Dohi K, Fujioka M, Iida H, Kida H, Kibe Y, Hattori N, Takeuchi J, (1978) Primary IgA glomerulonephritis and Schönlein-Henoch purpura nephritis: Clinicopathological and immunohistological characteristics. Q J Med 47: 495-516

28. Davin JC, Ten Berge IJ, Weening JJ, (2001) What is the difference between $\operatorname{IgA}$ nephropathy and Henoch-Schönlein purpura nephritis? Kidney Int 59: 823-834

29. Droz D, Lantz B (1995) Biopsie rénale. Inserm, Paris, 151-153

30. Lv J, Shi S, Xu D, Zhang H, Troyanov S, Cattran DC, Wang H, (2013) Evaluation of the Oxford Classification of IgA nephropathy: a systematic review and meta-analysis. Am J Kidney Dis 62: 891-899

31. Herzenberg AM, Fogo AB, Reich HN, Troyanov S, Bavbek N, Massat AE, Hunley TE, Hladunewich MA, Julian BA, Fervenza FC, Cattran DC, (2011) Validation of the Oxford classification of IgA nephropathy. Kidney Int 80: 310-317 
32. Podjasek JO, Wetter DA, Pittelkow MR, Wada DA, (2012) Henoch-Schönlein purpura associated with solid-organ malignancies: three case reports and a literature review. Acta Derm Venereol 92: 388-392

33. Nadrous HF, Yu AC, Specks U, Ryu JH, (2004) Pulmonary involvement in Henoch-Schönlein purpura. Mayo Clin Proc 79: $1151-1157$

34. Narchi H, (2005) Risk of long term renal impairment and duration of follow up recommended for Henoch-Schönlein purpura with normal or minimal urinary findings: a systematic review. Arch Dis Child 90: 916-920

35. Hahn D, Hodson EM, Willis NS, Craig JC, (2015) Interventions for preventing and treating kidney disease in Henoch-Schönlein purpura (HSP) (Review). Cochrane Database Syst Rev 8: CD005128 (doi: 10.1002/14651858.CD005128.pub3)

36. Kawasaki Y, Suyama K, Hashimoto K, Hosoya M, (2011) Methylprednisolone pulse plus mizoribine in children with HenochSchoenlein purpura nephritis. Clin Rheumatol 30: 529-535

37. Niaudet P, Habib R, (1998) Methylprednisolone pulse therapy in the treatment of severe forms of Schönlein-Henoch purpura nephritis. Pediatr Nephrol 12: 238-243

38. Pillebout E, Alberti C, Guillevin L, Ouslimani A, Thervet E, (2010) Addition of cyclophosphamide to steroids provides no benefit compared with steroids alone in treating adult patients with severe Henoch Schönlein Purpura. Kidney Int 78: 495-502

39. Maritati F, Fenoglio R, Pillebout E, Emmi G, Urban ML, Rocco $\mathrm{R}$, Nicastro $\mathrm{M}$, Incerti M, Goldoni M, Trivioli G, Silvestri E, Mohammad AJ, Jayne D, Eriksson P, Segelmark M, Novikov P, Harris H, Roccatello D, Vaglio A, (2017) Brief report: rituximab for the treatment of adult-onset IgA vasculitis (HenochSchönlein). Arthritis Rheumatol 70: 109-114

40. group Kw, (2012) Chapter 11: Henoch-Schönlein purpura. Kidney Int Suppl 2: 218-220

41. Cherqaoui B, Chausset A, Stephan JL, Merlin E, (2016) Intravenous immunoglobulins for severe gastrointestinal involvement in pediatric Henoch-Schönlein purpura: a French retrospective study. Arch Pediatr 23: 584-590

42. Donghi D, Schanz U, Sahrbacher U, Recher M, Trueb RM, Mullhaupt B, French LE, Hafner J, (2009) Life-threatening or organimpairing Henoch-Schönlein purpura: plasmapheresis may save lives and limit organ damage. Dermatology 219: 167-170

43. Chaudhary K, Shin JY, Saab G, Luger AM, (2008) Successful treatment of Henoch-Schönlein purpura nephritis with plasma exchange in an adult male. NDT Plus 1: 303-306

44. Shenoy M, Bradbury MG, Lewis MA, Webb NJ, (2007) Outcome of Henoch-Schönlein purpura nephritis treated with longterm immunosuppression. Pediatr Nephrol 22: 1717-1722 